\title{
Hype, hyperscanning and embodied social neuroscience
}

\author{
Antonia Hamilton \\ Institute of Cognitive Neuroscience, UCL
}

\begin{abstract}
Hyperscanning has been hailed as a game-changing method which will allow us to understand the neuroscience of multi-person social interactions and create 'second person neuroscience'. Here, I present a critical review of fNIRS hyperscanning studies, examining what they can and cannot tell us about social neuroscience. A key problem is that many current hyperscanning methods cannot distinguish a pure pattern of interpersonal coherence from effects driven by a common input. Limited data on participant behaviour during testing sessions compounds this problem, because it is not clear what behaviours might mediate the brain coherence patterns that are reported. Some studies respond to this problem by retreating from strong cognitive interpretations of hyperscanning data and measuring how overall levels of coherence differ with individual factors (such as age, gender, social relationships between people or clinical diagnosis). Here, I suggest that there is a better way to analyse and interpret hyperscanning studies. By tracking behaviour in detail, and analysing behaviour and brain activity patterns together, it will be possible to define what types of action, perception and mutual prediction arising in the interaction of two or more people can lead to coherent brain signals and why. This approach acknowledges that social brains are embodied and that coherent brain activity arises from the social behaviour of two people in an interaction. By integrating our study of the social brain and social behaviour, we will be able to strengthen the science of both.
\end{abstract}


Neuroimaging methods have transformed our understanding of human cognition over the last 30 years. In 2002, the term 'hyperscanning' was introduced to describe situations in which two (or more) people participate in a neuroimaging study at the same time in an interactive context, and where data is analysed across-brains (Montague et al., 2002). The pioneering study from King-Casas et al used fMRI to record brain activity while participants interacted via an economic game (KingCasas et al., 2005), but more recently, hyperscanning has emerged as a popular approach in conjunction with EEG (Dikker et al., 2017) and with fNIRS (Pinti et al., 2020). These modalities allow participants to engage in face-to-face interactions and natural behaviours without the isolation of the MRI scanner. The present paper focuses on hyperscanning studies using fNIRS and examines what these can tell us about human social cognition.

Since the first NIRS hyperscanning study (Cui et al., 2012), over 80 data papers have been published and this area is growing rapidly. Many early papers provide a proof-of-principle that hyperscanning can be performed, and explore different methods for analysing the data (see below). It is now clear that fNIRS hyperscanning is feasible and can yield interesting data. However, there are still important questions which must be considered for the development of the field. The aim of the present paper is to examine the key challenges facing the development of hyperscanning, both in terms of methods and in terms of interpreting the data appropriately. This review aims to take a cautious approach, identifying the challenges so that we can tackle them head-on and find appropriate, feasible solutions so that we can get the best out of hyperscanning. First, we briefly consider why hyperscanning attracts so much interest, then review the approaches taken in major hyperscanning papers. We then consider what we can and cannot learn using this method, as well as considering some alternative ways to use fNIRS to study dynamic social interactions.

\section{Why should we do hyperscanning?}

Purchasing multiple recording devices, recruiting multiple participants and collecting hyperscanning data is a substantial piece of work, so it is first important to consider why this domain has attracted so much attention. One of the main reasons for the rise in hyperscanning can probably be seen in calls for the development of 'second person neuroscience' (Schilbach et al., 2013). This is the idea that researchers must move beyond studying social cognition in a single individual and attempt to understand the social brain in interactive contexts. A number of papers now make the claim that studying two people can tell us more than studying one person, because interaction is central to social cognition (De Jaegher et al., 2010) and that there are features that emerge in two person interactions that cannot be studied in traditional contexts (Gvirts \& Perlmutter, 2019; Hari et al., 2015; Hasson \& Frith, 2016; Redcay \& Schilbach, 2019).

It is clearly true that there are behaviours which emerge when two people perform together which cannot be studied with a single participant alone in a lab. These include mutual adaptation in generating rhythmic actions (Konvalinka et al., 2010), audience effects (Hamilton \& Lind, 2016) which arise when people respond differently to being watched by a person (Myllyneva \& Hietanen, 2015), joint action when two people perform a task together (Sebanz et al., 2006) and communicative dynamics (Fusaroli et al., 2012). Similarly, we know that different cognitive and neural mechanisms are engaged when participants believe they are interacting with a real human compared to a computer (Gallagher et al., 2002) and when they are being watched by another person (Izuma et al., 2010; Müller-Pinzler et al., 2015). Given these changes in the neurocognitive systems of a single person engaged in social interaction, it seems a logical next step that neuroimaging of both partners in a two person social interaction should tell us more than just studying one person at a time. The present paper examines this idea in more detail. 
Here it is important to make the distinction between analysing one-brain in a two person context and analysing two brains together (Redcay \& Schilbach, 2019). In the former case, we might invite two participants to the lab, even record from both of them simultaneously, but the data analyses use traditional one-person methods (e.g. a GLM which predicts brain activity based on specific events) and the conclusions which are drawn apply only to a single brain. Excellent examples of this can be seen in EEG work (Kourtis et al., 2013; Westley et al., 2017) and fMRI studies where the interaction partner is outside the scanner (Hampton et al., 2008).

In contrast, hyperscanning studies collect data from two (or more) participants simultaneously and analyse that data in a way that integrates the signals across participants. Such studies might measure the coherence in brain activity between the two brains, often using crosscorrelation or wavelet coherence methods (see below). The results of such analyses show us which brain regions have similar patterns of activity across two participants, and the outcome measure is a quantification of interpersonal neural synchrony (INS). Here, we will use the term hyperscanningINS or h-INS to specifically refer to measures of coherent neural activity collected from two or more people in an online interactive context (in contrast to sequential measures described below).

\section{A brief review of fNIRS hyperscanning}

Since the first fNIRS hyperscanning paper in 2012, over 80 papers have been published using this approach covering a range of methods. Here we briefly summarise some of the most influential papers in this area, grouped by the experimental paradigm used. The first fNIRS hyperscanning study published (Cui et al., 2012) gave participants a computerised cooperation / competition task where they must coordinate the timings of their actions in the cooperation condition. Signals were recorded from prefrontal cortex and analysed using wavelet coherence to show more h-INS was found in the cooperation condition compared to competition. A number of studies have now replicated this with larger samples and additional contrasts, testing for effects of visual feedback and participant relationships (Pan et al., 2017; Reindl et al., 2018). For example, Baker and colleagues collected data from 111 dyads to test for sex differences in INS, finding more coherence in same-sex dyads than in mixed-sex dyads (Baker et al., 2016). These studies suggest that different patterns of social relationship between people can lead to different levels of INS.

A different method is to manipulate the level of interaction between two people more explicitly. For example, there is more wavelet coherence between two people who talk face to face compared to talking back-to-back (Jiang et al., 2012) and between two people who sing or hum face to face compared to back-to-back (Osaka et al., 2015). In a series of carefully controlled studies, Hirsch et al have shown more coherence between two people making eye contact compared to looking at a picture (Hirsch et al., 2017) and between two people talking in dialogue compared to monologue (Hirsch et al., 2018). All these studies suggest that increasing the potential for a dynamic interaction between two people increases the wavelet coherence between their brains.

Other groups have used tasks which involve a more dynamic face to face interaction. Jiang et al studied conversational dynamics in groups of three people to see if INS related to the leadership role that participants adopted. Higher wavelet coherence in prefrontal cortex was found between leader-follower pairs compared to follower-follower pairs (Jiang, Chen, Dai, Shi, Ding, Liu, Lu, et al., 2015). Again using a multi-person approach, Fishburn et al asked participants to complete tangram puzzles in groups of 3 and found more coherence in prefrontal cortex between a pair of participants working together on the same puzzle, compared to the third person in the room who was working alone (Fishburn et al., 2018). Similarly, Nozawa et al studied a 4 person interaction where participants performed a cooperative word-chain task (Nozawa et al., 2016) and found 
coherence in prefrontal cortex. In all these multi-person studies, the involvement of more than two people might allow us to test if $h$-INS involves more than a shared reaction to a common environment (see below).

fNIRS hyperscanning can also be used as a measure of the success of a social interaction. For example, a study of pairs of participants showed that performing synchronous movements before an interaction lead to more h-INS signal in prefrontal cortex and more rapport but no increase in word learning (Nozawa et al., 2019). In another study of song learning, fNIRS was recorded from prefrontal cortex of teachers and learners. INS in the dyad predicted how well the learner acquired the song, and granger causality analyses showed that the teachers brain activity predicted that in the learner (Pan et al., 2018). Recently, a large scale study tested how a social bonding conversation impacts on performance in a group decision making game. Data was recorded from groups of six participants simultaneously, who were playing a attack-defence game where one group of three can coordinate to attack the other group of three, who collectively defend themselves. INS within each team was recorded from dIPFC and TPJ, and results showed that social bonding at the start of the session leads to increased INS in these brain systems and to more within-group cooperation and more between-group hostility (Yang et al., 2020).

In these studies, the majority use wavelet analyses to identify h-INS (Cui et al., 2012) but some use granger causality (Pan et al., 2018) or cross-brain correlations (Fishburn et al., 2018). Studies also vary substantially in the sample sizes reported (from 6 to over 100 dyads) and in the statistical methods. The precise algorithms used to calculate measures of coherence and the approaches to normalising data across participants and correcting results for multiple comparisons are also quite variable across labs and across studies. More work may be needed to standardise these analysis steps and increase the reproducibility of results across the field.

In the same time period, over 30 review papers \& theory papers have been published which make broad claims about the benefits of hyperscanning. However, the interpretation of hINS remains complex. At a basic level, a robust finding of hINS tells us that the brain of participant $A$ and the brain of participant $B$ have approximately the same pattern of activation at the same time. How we can interpret this in terms of cognition is less clear. In some review papers, rich interpersonal interpretations are given to hyperscanning methods. For example, brain-to-brain coupling has been described as a 'mechanism for transmitting information .... regarding remote events' (Hasson \& Frith, 2016) or as a 'mechanism of shared intentionality' (Fishburn et al., 2018). One paper even suggests that 'IBS might trigger the neural mechanism guiding social alignment' (Gvirts \& Perlmutter, 2019). Similarly, a recent review on EEG hyperscanning suggests that the entrainment between interacting brains might contribute to early learning (Wass et al., 2020). It has been suggested that hyperscanning measures might allow us to diagnose developmental and psychiatric disorders (Leong \& Schilbach, 2019) and that using brain stimulation to impose INS on participants might even treat such disorders (Gvirts \& Perlmutter, 2019). These are ambitious claims for an emerging technology, and deserve careful and detailed examination. 


\section{Sequential intersubject correlations and the problem of common input}

As a starting point for interpreting hINS, it is useful to consider a closely related finding inter-subject correlations in $\mathrm{fMRI}$ data which arise even when two participants are not interacting. In a landmark study for the neuroscience of natural stimuli, Hasson (Hasson et al., 2004) showed individual participants in an MRI scanner a classic movie (The Good, the Bad \& the Ugly) and calculated the correlation between the brain activation patterns across participants. Robust intersubject correlations in these sequential brain imaging studies were found across many visual and semantic areas, reflecting participant's consistent responses to the rich auditory-visual stimulus. Following the acronyms used by the Hasson lab, we refer to this signal as sequential inter-subject correlation (s-ISC). More recent studies using these measures of s-ISC show that it is stronger for more engaging movies (Hasson \& Landesman, 2008) and when participants have a similar interpretation of the movie (Nguyen et al., 2019). s-ISC can be seen when the same story is told in different languages (Yeshurun et al., 2017) and between a speaker and a listener (Stephens et al., 2010).

These results using sequential scanning methods, where there is no interaction between participants, show that there are robust and meaningful patterns of s-ISC in many contexts. Because these studies involve sequential scanning with a well-defined auditory-visual input, we can give a cognitive interpretation to the data. It seems that these s-ISC results are driven by a combination of the external auditory-visual environment and the participant's conceptual understanding of the story. That it, common visual input, common auditory in put and common conceptual understanding can drive s-ISC, even in the absence of any interaction between participants.

This is critical to interpreting hyperscanning studies because, in any fNIRS hyperscanning task, the two participants in the same room at the same time receive many common inputs: they hear the same words or see the same images on the screen or even feel the same rumbling as a large truck drives past the lab. This means it is very hard to know if the h-INS signal recorded in a hyperscanning study is just the result of common sensory inputs and is identical to s-ISC, or if h-INS is different and can give us something more. This is termed 'the problem of common input' and has also been identified in EEG hyperscanning (Burgess, 2013). One attempt to control for this issue is to analyse true pairs compared to pseudo pairs. Here, researchers contrast the level of coherence obtained for a dyad tested together to the level of coherence obtained for an 'artificial dyad' created by matching up the signals from two participants who completed the task but not at the same time. Finding of strong INS in the true pairs compared to pseudopairs could be evidence that the coherence signal reflects properties of the interaction itself and not of the task structure. However, this approach only works if the true pairs and pseudo pairs are very closely matched in the visual, auditory and motor events they experience. If a true pair has a conversation about cats while a pseudo pair talks about dogs (with different word timings etc), then differences in INS between them could be due to the behavioural differences. One approach to this problem is to impose tight behavioural constraint on the participants, such as required eye contact in

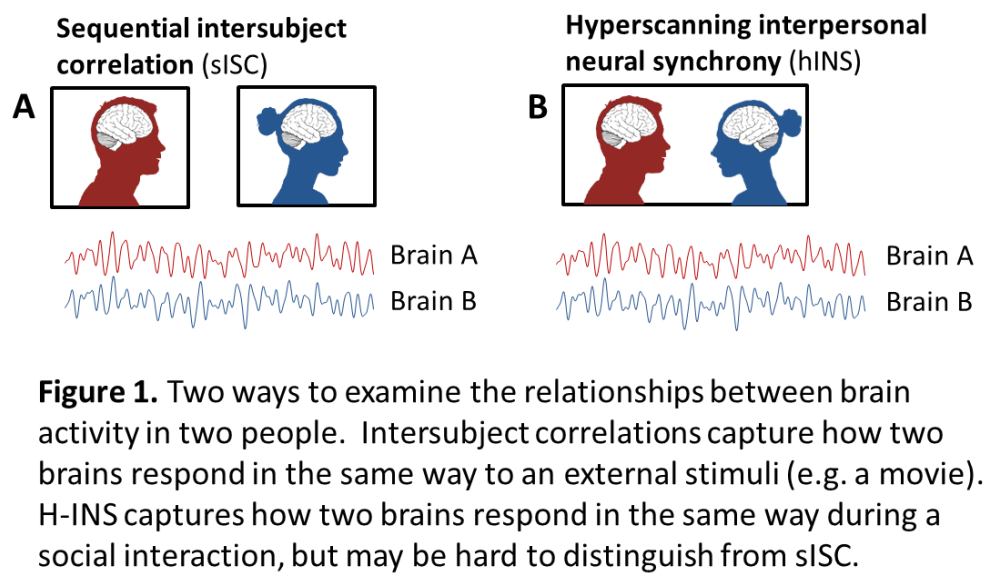


a sequence of three seconds on - three seconds off repeated for each trial (Hirsch et al., 2017). Such approaches yield data that are easier to interpret, but risk restricting the natural social behaviour which we are trying to study.

An alternative way to control for the problem of common input is to scan 3 participants or more rather than two. The logic of this method is that if $A, B$ and $C$ are in the same room experiencing the same sequence of events, but $A-B$ show higher $h-I N S$ than $A-C$ or $C-B$, then there must be something distinctive in the $A-B$ coherence beyond the common environment. This method was used to good effect in a landmark study of Jiang et al where trios of participants had conversations and greater coherence was found between the person who took the lead role in the conversation and the followers, compared to coherence between the followers (Jiang, Chen, Dai, Shi, Ding, Liu, \& Lu, 2015). Another example is a recent paper where trios of participants completed tangram puzzles in pairs or alone and more coherence was found between participants in a pair than between a paired worker and a lone worker (Fishburn et al., 2018). One limitation of both these studies is that there is only limited data on the motor behaviour of the participants, and it is likely that the two people working together coordinated their movements in time, while the solo participant did not. Thus, it is not easy to rule out the possibility that this effect is driven by the interacting participants having a common motor environment which would impact on the data in the same way as a common sensory environment.

To summarise, the problems of common input provides a significant challenge to the interpretation of fNIRS hyperscanning studies. Innovative approaches are being used to tackle this issue, but it is not yet clear if any of them can eliminate the problem. In the following sections, we suggest here that there may be other ways to understand and used hyperscanning methods, which might skirt around the issue and be fruitful in the long term. First, there is the option to take a weak, deflationary interpretation of h-INS data in which common inputs don't really matter, and to see if this is still helpful in advancing social neuroscience. Second, there is the option to build on our strong knowledge of how to do single-brain analyses of neuroimaging data and to apply these more widely in a hyperscanning context.

\section{INS as a proxy for 'thinking the same'}

The brief review above highlights how the interpretation of h-INS data is not straightforward. It is still early days in this field, and future studies with careful designs may yet reveal robust components in the h-INS signal which cannot be attributed solely to common inputs or common outputs. However, it is still useful to think about simpler interpretations of our data. One deflationary option is to argue that the distinction between hyperscanning INS and sequential ISC is not terribly important, and that both can index the same thing, that is, both signals could provide a general index of 'how much two brains interpret events in the same way'. Emerging evidence suggests that sISC indexes not just the auditory-visual input but also the cognitive interpretation participants place on that input (Nguyen et al., 2019; Stephens et al., 2010). If h-INS also indexes a shared cognitive interpretation of the world, then finding INS in some dyads but not others might tell us something interesting about those dyads. For example, the level of h-INS between clients and therapists is larger in pairs with a stronger working alliance (Zhang et al., 2018) and thus might index how well these pairs can work together. Similarly, the level of INS in group decision making seems to predict with-group coordination and between-group hostility (REF - Yang 2020). Used in this way, INS could provide a measure of whether two people converge or diverge in their interpretation of events as a conversation progresses, or as a proxy for the closeness of their conversation. 
Interpreting the h-INS signal in this simple fashion, as an index of 'thinking the same' makes it much easier to draw conclusions from hyperscanning studies, and to potentially used $h$-INS as a clinical measure or as a way to study individual differences in social cognition (Leong \& Schilbach, 2019). However, there are still some challenges for this interpretation. First, if we accept that h-INS is largely the same as s-ISC and that both signals index 'thinking the same', then we might ask why researchers should bother to use hyperscanning rather than sequential scanning methods. The latter gives better experimental control and repeatability which might be more suitable for patient populations or for the study of individual differences. Second, developing a very general measure of how much people 'think the same' and using it across populations runs the risk that researchers will expect and value social conformity. That is, it might be practical to develop a measure which tells us much any one person's pattern of brain activity conforms to the 'typical' activity pattern defined by another group of people. However, we should consider carefully who contributes to the control group that defines 'typical' activity patterns and whether these are WEIRD participants (Henrich et al., 2010). It would not be good to develop neural measures that assume there should always be conformity between different groups of people who have different ways of interpreting the world. These caveats mean that the deflationary account of h-INS might be a useful starting point, but that it would be helpful to go beyond this.

\section{The mutual prediction account of h-INS}

An important advance in our analysis and interpretation of hyperscanning data has arrived with the use of recordings from prefrontal cortical neurons in pairs of interacting rats (Kingsbury et al., 2019). In this paper, the activity patterns of prefrontal cortex in pairs of interacting rats were recorded with calcium imaging while the animals could explore the same cage or meet in a narrow tube (a competitive situation). Global activity levels recorded across prefrontal cortex were correlated between the two rats, and this correlation was greater when the animals were in the same space and were interacting. To understand the signals in more detail, the paper used a general linear model, similar to classical fMRI analysis methods, to model the brain activity of animal $A$, based on regressors describing the behaviour of $A$, the behaviour of $B$ and the brain activity of $B$. Including the brain activity of $B$ in the model improved the model fit, and this was particularly true when there was a clear dominant-subordinate relationship between the two rats. Further detailed coding of the rat behaviour revealed that PFC for each rat had both neurons selective to the rat's own behaviour and those selective to the partner's behaviour, and that the behaviour of the dominant rat was the strongest predictor of interbrain synchrony. The authors interpret this data in terms of a mutual prediction model of hyperscanning, in which both individuals are predicting the behaviour of their partner at the same time, and this gives rise to coherent signals across both individuals.

These studies have two important implications for future research on human social interaction using hyperscanning methods. First, if we apply a mutual prediction account to human behaviour, this predicts the involvement of the human mirror neuron system (premotor cortex and parietal cortex) in driving interpersonal brain synchrony, at least for movement tasks. The MNS is part of the visual motor network and is known to respond when people view other people's actions, perform hand actions and imitate hand actions (Caspers et al., 2010). It has a key role in predicting actions (Kilner et al., 2007) and in preparing responses to other people's actions (Hamilton, 2016; Sartori et al., 2013). This means that it has all the properties required to generate mutual predictions of self and other, and to coordinate these during social interaction. Few fNIRS studies so far have recorded from MNS regions, because many have focused on prefrontal cortex (where there is less hair), but both the IFG and IPL are accessible to fNIRS and could be a target for future studies. 
Second,

understanding mutual

prediction in interaction

will require detailed

knowledge of actions

themselves. That is,

kinematic recordings of

how people behave in an

interaction, together with

eyetracking and detailed

transcripts and coding of

speech, will be critical to

make sense of

hyperscanning data. Such

behavioural detail will
Embodied Social Neuroscience

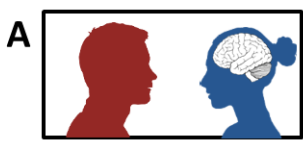

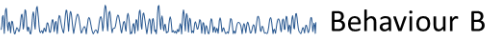

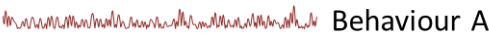
$\square \neg \square \_$Task

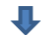
wollywhyngumm Brain B

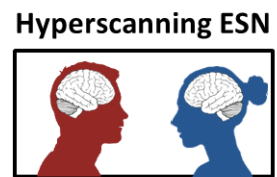

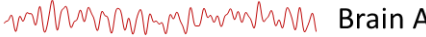

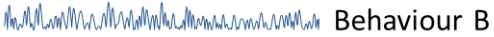

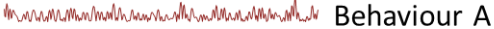
$\square \_-\neg \longrightarrow$ Task

7

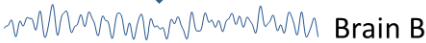

Figure 2. The Embodied social neuroscience approach records the behavior of both participants in detail in order to build a model predicting the brain activity in one participant. In the Hyperscanning ESN approach, this extends to using brain activity patterns recorded from participant $A$ to predict the brain activity in participant $B$.

allow us to go beyond generalities about how two brains respond the same, and to track in higher resolution how each participant responds to their partner. Related to this, it is important to note that the major conclusions of Kingsbury et al derive not from their analyses of interbrain coherence, but rather from applying traditional single-brain methods to data recorded from two brains at once. That is, Kingsbury and colleagues model the activity of a single brain with a GLM (similar to traditional fMRI methods) and include predictors based on both detailed behaviour coding and on the brain activity of the interaction partner.

Recent studies in our own lab draw on a similar approach to understanding human social interaction. For example, in Pinti et al, pairs of participants played a card game together where each round gave one participant the chance to lie (or tell the truth) and the other had to guess if their partner lied or not. fNIRS signals were recorded from 22 channels across PFC during the task. Traditional GLM analyses could identify brain regions active when lying and when detecting lies. In addition, a cross-brain model which used the signal from PFC of the task leader to predict the brain activity of the follower identified optodes in medial PFC with a 2 second delay between the two brains. This implies that the follower's brain is echoing the leaders, in line with the information structure of the task.

In a second study, (Cañigueral, Zhang, Noah, Tachtsidis, Hamilton \& Hirsch, in prep.), pairs of participants sat across a table facing each other while they indicated their personal preferences or behaviours in different everyday situations (e.g. "I try not to cover up my mistakes"). In some blocks, their responses were shared with the partner (public blocks) but in others they were not (private blocks). During the task, participants were simultaneously recorded with eye-tracking, facetracking and functional near-infrared spectroscopy (fNIRS) to test how social behaviours (eye gaze and facial displays) and brain activity are modulated when sharing biographical information. Results showed that information sharing impacted on both social behaviour and brain activity. For instance, participants gazed more at each other's face and produced more facial displays during public trials, and this was associated within increased signal in temporoparietal junction (linked to mentalising) and dorsolateral PFC (linked to strategic decision making). Because detailed behavioural recordings were collected, in this study we could also model how the brain activity of each participant is influenced by that person's performed facial actions and by their partner's facial actions. We found that spontaneous production of facial displays (i.e. participants moving their own face) recruited the left supramarginal gyrus, whereas spontaneous observation of facial displays (i.e. participants seeing their partner move the face) recruited the right dorsolateral prefrontal cortex. This reveals the 
dynamic interplay of social signalling behaviours and brain activity patterns during live face to face interactions.

Note again that the primary analysis here focuses on the activation patterns in a single brain, using a fairly traditional GLM approach. By analysing natural behaviour of two socially interacting people in relation to brain activity patterns, we can form new insights into the mechanisms of social interaction. This does not rely on hyperscanning alone, but rather leverages the face-to-face context to understand embodied social signalling. Further analyses on the same dataset could test for the existence of hISC signals, but these are not the primary outcome.

\section{The embodied social neuroscience approach}

In the review above, we have described current approaches to human hyperscanning studies, and identified a number of challenges with this. In particular, the most common wavelet coherence measures can be confounded by the problem of common environment, may fail to pick up patterns of reciprocal interaction and they are hard to interpret beyond an idea of social conformity. We have also reviewed some alternative approaches, implemented both in rats and humans, which avoid some of these problems. In this final section, I aim to formalise this approach and use it to set out a more positive vision of what we can do with hyperscanning and how realworld neuroimaging can unlock important facets of social neuroscience.

The key step here is the recognition that any hINS is mediated by behaviour. Interpersonal neural synchrony is not a telepathic connection between one brain and another, but rather one person's brain activity causes a social behaviour - a smile or gaze or word - which is then perceived by the other person and causes a change in their brain. The physical body provides the medium through which hINS arises, and thus our study of social INS must be closely linked to our study of embodied and enactive cognition. There has been an increased interest in embodied cognition in the last decade and a recognition that the physical state of the body feeds back to and influences our cognitive state (Wilson, 2002). Similarly, the enactive cognition approach focuses on how we implement our cognitions with our body and in interaction with the physical world (Thompson \& Varela, 2001).

Here, I suggest that we should place our understanding of second person neuroscience within an embodied and enactive framework where we must understand brains in bodies. This leads to two outcomes. First, when we study hyperscanning, it is not enough just to record brain signals; it is critical to also record the body. This includes physiological signals (heart beat, breathing) which can impact on the fNIRS signal (Tachtsidis \& Scholkmann, 2016) and behaviour such as speech, gaze, facial movement and hand movement. An increasing number of motion capture technologies mean make this feasible, including wearable eyetrackers (Kredel et al., 2017) analysis of facial motion with computer vision (Baltrusaitis et al., 2016) and sensitive movement tracking (Hale et al., 2020; Pishchulin et al., 2016). Such systems could be standard in a hyperscanning lab.

There are several benefits to this embodied social neuroscience approach. First, this strategy avoid the pitfalls and challenges to h-INS which have been detailed above and provides a new way to move forward. Second, because we analyse data from one brain at a time, it is easier to build on existing analysis methods (e.g. SPM) and existing cognitive models of non-social brain systems, with the potential for a stronger integration of the ESC approach with our current models of cognitive neuroscience. There is potential to identify clear \& specific mechanisms, and to use this in conjunction with many different behaviours \& groups. Finally, ESC can lead to a strong understanding of the roles brain and body play together in social interaction, describing how the 
meeting of minds is mediated by an interaction of bodies. Studying two people in a genuine interaction is essential here, because people don't have the same behaviours \& cognitions when alone. But we recognise that that computations that drive behaviour still happen within a single brain and can be analysed within a single brain.

\section{Conclusions}

This paper aims to sound a note of caution on hyperscanning studies, but also to understand how they can be used and what are the alternatives for studying interactive social neuroscience. Our review suggests that h-ISC signals can be used as a proxy for 'similar processing' in the same vein as s-INS, but should not be described as a 'mechanism'. We further suggest that understanding how social interactions are embodied and the relationship between social behaviours and the social brain in the context of interaction may be a fruitful direction for further study of human interaction.

\section{References}

Baker, J. M., Liu, N., Cui, X., Vrticka, P., Saggar, M., Hosseini, S. M. H., \& Reiss, A. L. (2016). Sex differences in neural and behavioral signatures of cooperation revealed by fNIRS hyperscanning. Scientific Reports, 6(1), 26492. https://doi.org/10.1038/srep26492

Baltrusaitis, T., Robinson, P., \& Morency, L.-P. (2016). OpenFace: An open source facial behavior analysis toolkit. 2016 IEEE Winter Conference on Applications of Computer Vision (WACV), 1-10. https://doi.org/10.1109/WACV.2016.7477553

Burgess, A. P. (2013). On the interpretation of synchronization in EEG hyperscanning studies: a cautionary note. Frontiers in Human Neuroscience, 7, 881.

https://doi.org/10.3389/fnhum.2013.00881

Caspers, S., Zilles, K., Laird, A. R., \& Eickhoff, S. B. (2010). ALE meta-analysis of action observation and imitation in the human brain. In Neurolmage (2010/01/09, Vol. 50, Issue 3). Elsevier B.V. https://doi.org/10.1016/j.neuroimage.2009.12.112

Cui, X., Bryant, D. M., \& Reiss, A. L. (2012). NIRS-based hyperscanning reveals increased interpersonal coherence in superior frontal cortex during cooperation. Neurolmage, 59(3), 2430-2437. https://doi.org/10.1016/j.neuroimage.2011.09.003

De Jaegher, H., Di Paolo, E., \& Gallagher, S. (2010). Can social interaction constitute social cognition? Trends in Cognitive Sciences, 14(10), 441-447. https://doi.org/10.1016/j.tics.2010.06.009

Dikker, S., Wan, L., Davidesco, I., Kaggen, L., Oostrik, M., McClintock, J., Rowland, J., Michalareas, G., Van Bavel, J. J., Ding, M., \& Poeppel, D. (2017). Brain-to-Brain Synchrony Tracks Real-World Dynamic Group Interactions in the Classroom. Current Biology, 27(9), 1375-1380. https://doi.org/10.1016/j.cub.2017.04.002

Fishburn, F. A., Murty, V. P., Hlutkowsky, C. O., MacGillivray, C. E., Bemis, L. M., Murphy, M. E., Huppert, T. J., \& Perlman, S. B. (2018). Putting our heads together: interpersonal neural synchronization as a biological mechanism for shared intentionality. Social Cognitive and Affective Neuroscience, 13(8), 841-849. https://doi.org/10.1093/scan/nsy060

Fusaroli, R., Bahrami, B., Olsen, K., Roepstorff, A., Rees, G., Frith, C. D., \& Tylén, K. (2012). Coming to Terms: Quantifying the Benefits of Linguistic Coordination. Psychological Science, 23(July), 931939. https://doi.org/10.1177/0956797612436816

Gallagher, H. L., Jack, A. I., Roepstorff, A., \& Frith, C. D. (2002). Imaging the intentional stance in a 
competitive game. Neuroimage, 16(3 Pt 1), 814-21. https://doi.org/10.1006/nimg.2002.1117

Gvirts, H. Z., \& Perlmutter, R. (2019). What Guides Us to Neurally and Behaviorally Align With Anyone Specific? A Neurobiological Model Based on fNIRS Hyperscanning Studies. The Neuroscientist, 107385841986191. https://doi.org/10.1177/1073858419861912

Hale, J., Ward, J., Buccheri, F., Oliver, D., \& Hamilton, A. F. de C. (2020). Are you on my wavelength? Interpersonal coordination in dyadic conversations. Journal of Nonverbal Behaviour. http://www.antoniahamilton.com/HaleWard_JNVB_preprint.pdf

Hamilton, A. F. de C. (2016). The social function of the human mirror system: A motor chauvinist view. In S. S. Obhi \& E. S. Cross (Eds.), Shared Representations. Sensorimotor Foundations of Social Life (pp. 313-331). Cambridge University Press.

Hamilton, A. F. de C., \& Lind, F. (2016). Audience effects: what can they tell us about social neuroscience, theory of mind and autism? Culture and Brain, 4(2), 159-177. https://doi.org/10.1007/s40167-016-0044-5

Hampton, A. N., Bossaerts, P., \& O’Doherty, J. P. (2008). Neural correlates of mentalizing-related computations during strategic interactions in humans. Proceedings of the National Academy of Sciences of the United States of America, 105(18), 6741-6746. https://doi.org/10.1073/pnas.0711099105

Hari, R., Henriksson, L., Malinen, S., \& Parkkonen, L. (2015). Centrality of Social Interaction in Human Brain Function. Neuron, 88(1), 181-193. https://doi.org/10.1016/j.neuron.2015.09.022

Hasson, U., \& Frith, C. D. (2016). Mirroring and beyond: Coupled dynamics as a generalized framework for modelling social interactions. In Philosophical Transactions of the Royal Society B: Biological Sciences (Vol. 371, Issue 1693). https://doi.org/10.1098/rstb.2015.0366

Hasson, U., \& Landesman, O. (2008). Neurocinematics: The neuroscience of film. ..., 2(1), 1-26. http://ohadlandesman.com/pdf/Neurocinematics-Projections2008.pdf

Hasson, U., Nir, Y., Levy, I., Fuhrmann, G., \& Malach, R. (2004). Intersubject synchronization of cortical activity during natural vision. Science, 303(5664), 1634-1640.

https://doi.org/10.1126/science.1089506

Henrich, J., Heine, S. J., \& Norenzayan, A. (2010). Most people are not WEIRD. In Nature (Vol. 466, Issue 7302, p. 29). Nature Publishing Group. https://doi.org/10.1038/466029a

Hirsch, J., Adam Noah, J., Zhang, X., Dravida, S., \& Ono, Y. (2018). A cross-brain neural mechanism for human-to-human verbal communication. Social Cognitive and Affective Neuroscience, 13(9), 907-920. https://doi.org/10.1093/scan/nsy070

Hirsch, J., Zhang, X., Noah, J. A., \& Ono, Y. (2017). Frontal temporal and parietal systems synchronize within and across brains during live eye-to-eye contact. Neurolmage, 157, 314-330. https://doi.org/10.1016/j.neuroimage.2017.06.018

Izuma, K., Saito, D. N., \& Sadato, N. (2010). The roles of the medial prefrontal cortex and striatum in reputation processing. Social Neuroscience, 5(2), 133-147.

Jiang, J., Chen, C., Dai, B., Shi, G., Ding, G., Liu, L., \& Lu, C. (2015). Leader emergence through interpersonal neural synchronization. Proceedings of the National Academy of Sciences of the United States of America, 112(14), 4274-4279. https://doi.org/10.1073/pnas.1422930112

Jiang, J., Chen, C., Dai, B., Shi, G., Ding, G., Liu, L., Lu, C., \& Fiske, S. T. (2015). Leader emergence through interpersonal neural synchronization. Proceedings of the National Academy of Sciences of the United States of America, 112(14), 4274-4279. 
https://doi.org/10.1073/pnas.1422930112

Jiang, J., Dai, B., Peng, D., Zhu, C., Liu, L., \& Lu, C. (2012). Neural synchronization during face-to-face communication. Journal of Neuroscience, 32(45), 16064-16069.

https://doi.org/10.1523/JNEUROSCI.2926-12.2012

Kilner, J., Friston, K. J., \& Frith, C. D. (2007). Predictive coding: an account of the mirror neuron system. Cognitive Processing, 8(3), 159-166. https://doi.org/10.1007/s10339-007-0170-2

King-Casas, B., Tomlin, D., Anen, C., Camerer, C. F., Quartz, S. R., \& Montague, P. R. (2005). Getting to know you: reputation and trust in a two-person economic exchange. Science (New York, N.Y.), 308(5718), 78-83. https://doi.org/10.1126/science.1108062

Kingsbury, L., Huang, S., Wang, J., Gu, K., Golshani, P., Wu, Y. E., \& Hong, W. (2019). Correlated Neural Activity and Encoding of Behavior across Brains of Socially Interacting Animals. Cell. https://doi.org/10.1016/j.cell.2019.05.022

Konvalinka, I., Vuust, P., Roepstorff, A., \& Frith, C. D. (2010). Follow you, follow me: continuous mutual prediction and adaptation in joint tapping. Quarterly Journal of Experimental Psychology (2006), 63(11), 2220-2230. https://doi.org/10.1080/17470218.2010.497843

Kourtis, D., Sebanz, N., \& Knoblich, G. (2013). Predictive representation of other people's actions in joint action planning: An EEG study. SOCIAL NEUROSCIENCE, 8(1), 31-42. https://doi.org/10.1080/17470919.2012.694823

Kredel, R., Vater, C., Klostermann, A., \& Hossner, E.-J. (2017). Eye-Tracking Technology and the Dynamics of Natural Gaze Behavior in Sports: A Systematic Review of 40 Years of Research. Frontiers in Psychology, 8(OCT), 1845. https://doi.org/10.3389/fpsyg.2017.01845

Leong, V., \& Schilbach, L. (2019). The promise of two-person neuroscience for developmental psychiatry: Using interaction-based sociometrics to identify disorders of social interaction. In British Journal of Psychiatry (Vol. 215, Issue 5, pp. 636-638). Cambridge University Press. https://doi.org/10.1192/bjp.2019.73

Montague, P. R., Berns, G. S., Cohen, J. D., McClure, S. M., Pagnoni, G., Dhamala, M., Wiest, M. C., Karpov, I., King, R. D., Apple, N., \& Fisher, R. E. (2002). Hyperscanning: simultaneous fMRI during linked social interactions. Neuroimage, 16(4), 1159-64.

http://www.ncbi.nlm.nih.gov/htbin-post/Entrez/query?db=m\&form=6\&dopt=r\&uid=12202103

Müller-Pinzler, L., Gazzola, V., Keysers, C., Sommer, J., Jansen, A., Frässle, S., Einhäuser, W., Paulus, F. M., \& Krach, S. (2015). Neural pathways of embarrassment and their modulation by social anxiety. Neurolmage, 119, 252-261. https://doi.org/10.1016/j.neuroimage.2015.06.036

Myllyneva, A., \& Hietanen, J. K. (2015). The dual nature of eye contact: to see and to be seen. Social Cognitive and Affective Neuroscience, nsv075-. https://doi.org/10.1093/scan/nsv075

Nguyen, M., Vanderwal, T., \& Hasson, U. (2019). Shared understanding of narratives is correlated with shared neural responses. Neurolmage, 184, 161-170.

https://doi.org/10.1016/j.neuroimage.2018.09.010

Nozawa, T., Sakaki, K., Ikeda, S., Jeong, H., Yamazaki, S., Kawata, K. H. dos S., Kawata, N. Y. dos S., Sasaki, Y., Kulason, K., Hirano, K., Miyake, Y., \& Kawashima, R. (2019). Prior physical synchrony enhances rapport and inter-brain synchronization during subsequent educational communication. Scientific Reports, 9(1), 1-13. https://doi.org/10.1038/s41598-019-49257-z

Nozawa, T., Sasaki, Y., Sakaki, K., Yokoyama, R., \& Kawashima, R. (2016). Interpersonal frontopolar neural synchronization in group communication: An exploration toward fNIRS hyperscanning of 
natural interactions. Neurolmage, 133, 484-497.

https://doi.org/10.1016/J.NEUROIMAGE.2016.03.059

Osaka, N., Minamoto, T., Yaoi, K., Azuma, M., Shimada, Y. M., \& Osaka, M. (2015). How Two Brains Make One Synchronized Mind in the Inferior Frontal Cortex: fNIRS-Based Hyperscanning During Cooperative Singing. Frontiers in Psychology, 6, 1811. https://doi.org/10.3389/fpsyg.2015.01811

Pan, Y., Cheng, X., Zhang, Z., Li, X., \& Hu, Y. (2017). Cooperation in lovers: An fNIRS-based hyperscanning study. Human Brain Mapping, 38(2), 831-841. https://doi.org/10.1002/hbm.23421

Pan, Y., Novembre, G., Song, B., Li, X., \& Hu, Y. (2018). Interpersonal synchronization of inferior frontal cortices tracks social interactive learning of a song. Neurolmage, 183, 280-290. https://doi.org/10.1016/j.neuroimage.2018.08.005

Pinti, P., Tachtsidis, I., Hamilton, A., Hirsch, J., Aichelburg, C., Gilbert, S., \& Burgess, P. W. (2020). The present and future use of functional near-infrared spectroscopy (fNIRS) for cognitive neuroscience. Annals of the New York Academy of Sciences, 1464(1), 5-29. https://doi.org/10.1111/nyas.13948

Pishchulin, L., Insafutdinov, E., Tang, S., Andres, B., Andriluka, M., Gehler, P., \& Schiele, B. (2016). DeepCut: Joint Subset Partition and Labeling for Multi Person Pose Estimation. http://pose.mpiinf.mpg.de

Redcay, E., \& Schilbach, L. (2019). Using second-person neuroscience to elucidate the mechanisms of social interaction. Nature Reviews Neuroscience, 20(8), 495-505. https://doi.org/10.1038/s41583-019-0179-4

Reindl, V., Gerloff, C., Scharke, W., \& Konrad, K. (2018). Brain-to-brain synchrony in parent-child dyads and the relationship with emotion regulation revealed by fNIRS-based hyperscanning. Neurolmage, 178, 493-502. https://doi.org/10.1016/j.neuroimage.2018.05.060

Sartori, L., Bucchioni, G., \& Castiello, U. (2013). When emulation becomes reciprocity. Social Cognitive and Affective Neuroscience, 8(6), 662-669. https://doi.org/10.1093/scan/nss044

Schilbach, L., Timmermans, B., Reddy, V., Costall, A., Bente, G., Schlicht, T., \& Vogeley, K. (2013). Toward a second-person neuroscience. The Behavioral and Brain Sciences, 36(4), 393-414. https://doi.org/10.1017/S0140525X12000660

Sebanz, N., Bekkering, H., \& Knoblich, G. (2006). Joint action: bodies and minds moving together. Trends Cogn Sci, 10(2), 70-76. https://doi.org/S1364-6613(05)00356-6 [pii]10.1016/j.tics.2005.12.009

Stephens, G. J., Silbert, L. J., \& Hasson, U. (2010). Speaker-listener neural coupling underlies successful communication. Proceedings of the National Academy of Sciences. https://doi.org/10.1073/pnas.1008662107

Tachtsidis, I., \& Scholkmann, F. (2016). False positives and false negatives in functional near-infrared spectroscopy: issues, challenges, and the way forward. Neurophotonics, 3(3), 031405. https://doi.org/10.1117/1.NPh.3.3.031405

Thompson, E., \& Varela, F. J. (2001). Radical embodiment: Neural dynamics and consciousness. In Trends in Cognitive Sciences (Vol. 5, Issue 10, pp. 418-425). Elsevier Current Trends. https://doi.org/10.1016/\$1364-6613(00)01750-2

Wass, S. V., Whitehorn, M., Marriott Haresign, I., Phillips, E., \& Leong, V. (2020). Interpersonal Neural 
Entrainment during Early Social Interaction. Trends in Cognitive Sciences, 24(1), 1-14.

https://doi.org/10.1016/j.tics.2020.01.006

Westley, A., Kohút, Z., \& Rueschemeyer, S. A. (2017). “I know something you don't know”: Discourse and social context effects on the $\mathrm{N} 400$ in adolescents. Journal of Experimental Child Psychology, 164, 45-54. https://doi.org/10.1016/j.jecp.2017.06.016

Wilson, M. (2002). Six views of embodied cognition. Psychonomic Bulletin \& Review, 9(4), 625-636. https://doi.org/10.3758/BF03196322

Yang, J., Zhang, H., Ni, J., De Dreu, C. K. W., \& Ma, Y. (2020). Within-group synchronization in the prefrontal cortex associates with intergroup conflict. Nature Neuroscience, 1-7. https://doi.org/10.1038/s41593-020-0630-x

Yeshurun, Y., Swanson, S., Simony, E., Chen, J., Lazaridi, C., Honey, C. J., \& Hasson, U. (2017). Same Story, Different Story. Psychological Science, 095679761668202. https://doi.org/10.1177/0956797616682029

Zhang, Y., Meng, T., Hou, Y., Pan, Y., \& Hu, Y. (2018). Interpersonal brain synchronization associated with working alliance during psychological counseling. Psychiatry Research - Neuroimaging, 282, 103-109. https://doi.org/10.1016/j.pscychresns.2018.09.007 